Rev. Elev. Méd. Vét. Pays trop., 1976, 29 (2) . 95-99.

\title{
La fièvre aphteuse en Éthiopie Étude sérologique et immunologique d'une souche de virus aphteux de type A
}

\author{
par J. L. MARTEL (*) \\ (avec la collaboration technique de BEHRANU BEYENE $\left({ }^{* *}\right)$ )
}

\begin{abstract}
RÉSUMÉ
L'étude sérologique et immunologique de la souche A Gafarsa, isolée en Ethiopie au cours de l'épizootıe de 1974, montre qu'elle est différente des souches $\mathrm{A}$ isolées lors des précédentes épizooties. La couverture immunologique procuré par deux vaccins exotiques s'avère insuffisante pour protéger les bovins contre la souche A Gafarsa.
\end{abstract}

\section{INTRODUCTION}

L'étude sérologique comparative entre les différentes souches de virus aphteux de type A, isolées en Ethiopie de 1969 à 1974, a révélé une assez grande hétérogénéité au sein de ce type $(1,5)$.

Depuis février 1974, une nouvelle et importante épizootie de fièvre aphteuse de type $\mathbf{A}$ s'est manifestée dans le Shoa, Province centrale de l'Ethiopie, après une absence totale de manifestation de foyers de ce type pendant 4 années.

Parmi les souches isolées au cours de cette dernière épizootie, nous avons retenu tout particulièrement la souche isolée à Gafarsa en novembre 1974 sur des bovins laitiers régulièrement vaccinés contre la fièvre aphteuse avec un vaccin trivalent $\mathrm{OAC}$ importé d'Afrique Orientale.

L'étude sérologique et immunologique de cette souche fait l'objet de la présente note.

(*) Mission Vétérinaire Française en Ethiopie, P. O. Box 1053, Addis Ababa et Veterinary Institute, P. O. Box 19, Debré Zeit. Ethiopie.

(*) Gouvernement Ethiopıen. Ministère de l'Agriculture. Direction des Services vétérinaires.

\section{MATÉRIEL ET MÉTHODE}

\section{A. Etude sérologique}

Nous avons titré les anticorps antiaphteux produits sur des cobayes hyperimmunisés avec diverses souches de type A, par la technique de fixation du complément quantitative de semiprécision décrite dans une publication précédente (5).

Les titrages comparatifs des sérums hyperimmuns de type $\mathrm{A}$ déjà étudiés précédemment, réalisés d'une part vis-à-vis des antigènes homologues et d'autre part contre l'antigène $A$ Gafarsa, permettent de calculer une premiere série de relations sérologiques unilatérales $r_{2}$ entre la souche A Gafarsa et les autres souches à notre disposition.

De même, nous avons réalisé les titrages comparatifs du sérum anti A Gafarsa vis-à-vis du virus homologue d'une part et vis-à-vis des autres antigènes d'autre part. Ces titres nous permettent d'établir une deuxième série de relations sérologiques unilatérales $\mathrm{r}_{\mathrm{b}}$.

La moyenne géométrique de chaque couple de valeurs $r_{a} \cdot r_{b}$ exprime d'une manière chiffrée la parenté sérologique bilatérale. 


\section{B. Etude immunologique}

\section{1) Préparation du vaccin expérimental}

Nous avons préparé un vaccin expérimental avec la souche A Gafarsa. Le virus a été produit sur culture dite monocouche de cellules IBRS 2 en flacons roulants. Le nombre de passages a été réduit à 4 pour éviter au maximum d'éventuelles modifications du virus sauvage.

Le virus, récolté après $17 \mathrm{~h}$ de culture, est chloroformé et clarifié par centrifugation, puis adsorbé sur gel d’alumine et concentré de telle sorte que deux parties de virus brut fournissent une partie de vaccin terminé.

Le virus adsorbé et concentré est ensuite inactivé par l'effet conjoint du formol à $0,3 \mathrm{p}$. 1000 et de la chaleur $\left(30^{\circ} \mathrm{C}\right.$ pendant $36 \mathrm{~h})$.

Le vaccin est saponiné de façon qu'une dose bovine trivalente contienne $1 \mathrm{mg}$ de saponine. En fin de fabrication, le $\mathrm{pH}$ est stabilisé vers la valeur 8,2 par adjonction d'une quantité adéquate de tampon glycocolle.

Le vaccin est enfin soumis à un contrôle de stérilité sur milieux bactériologiques usuels et un contrôle d'innocuité sur souriceaux.

\section{2) Immunisation des bovins}

Nous avons choisi des bovins demi-sang Holstein X Zébu Local dépourvus d'anticorps neutralisants de type A.

Le vaccin monovalent A Gafarsa a été mélangé à 2 autres vaccins monovalents de type $\mathrm{O}$ et $\mathrm{C}$ préparés selon la même technique, ceci pour que les conditions d'immunisation soient analogues à celles de la pratique avec les vaccins commerciaux trivalents $\mathrm{OAC}$.

La dose bovine trivalente est de $5 \mathrm{ml}$, injectée par voie sous-cutanée.

Nous avons constitué, au hasard, 3 lots de 5 bovins chacun :

- le lot $n^{\circ} 1$ : immunisation avec une dose de vaccin expérimental de Debré Zeit ;

- le lot $n^{0} 2$ : immunisation avec une dose de vaccin commercial importé d'Europe ;

- le lot $n^{\circ} 3$ : immunisation avec une dose de vaccin commercial importé d'Afrique Orientale.

Ces 15 bovins ont été vaccinés le même jour et entretenus ensemble, dans les mêmes conditions, ainsi que 2 bovins témoins non vaccinés.

\section{3) Appréciation de l'immunité}

L'immunité a été appréciée par deux méthodes mises en œuvre simultanément, 21 jours après la vaccination.

a) Méthode directe : épreuve virulente.

Chaque bovin reçoit par voie intradermolinguale une dose fixe de virus A Gafarsa: 10000 doses infectieuses bovines 50 p. 100 en deux points de $0,1 \mathrm{ml}$ chacun.

Six jours plus tard, on note l'importance des lésions primaires éventuellement développées au niveau de la langue et les lésions secondaires de généralisation possibles au niveau des pieds.

Le critère de protection est l'absence totale de lésions de généralisation (6).

b) Méthode indirecte : titrage des anticorps sériques post-vaccinaux.

Le pouvoir neutralisant du sérum des animaux vaccinés est titré en mettant en présence des dilutions variables de chaque sérum et une quantité fixe de virus A Gafarsa.

Le système révélateur de la séroneutralisation est constitué par 5 tubes de culture de cellules IBRS 2 par dilution sérique. Chaque tube reçoit 50 doses infectieuses pour la culture cellulaire 50 p. 100.

On détermine le taux de la dilution de sérum qui protège le tapis cellulaire dans la moitié des tubes inoculés et on exprime les résultats sous la forme logarithmique $\mathrm{n}$ de l'indice de séroneutralisation $10^{\mathrm{n}}$ qui est l'inverse de la dilution neutralisante à 50 p. 100 (2).

\section{RÉSULTATS}

\section{A. Etude sérologique}

Le tableau $\mathrm{n}^{\circ} \mathrm{I}$ présente les parentés sérologiques bilatérales entre la souche A Gafarsa et les autres souches de même type calculées à partir des titres homologues et hétérologues des divers sérums hyperimmuns essayés.

\section{TABLEAU No I}

Parentés sérologiques bilatérales entre la souche A Gafarsa et les autres souches de type A

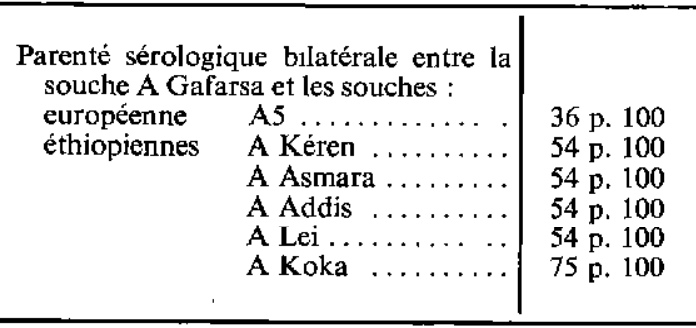


TABlEAL $\lambda^{\circ} I I$ - Étude innumulosqque de la souche A Gafarsa.

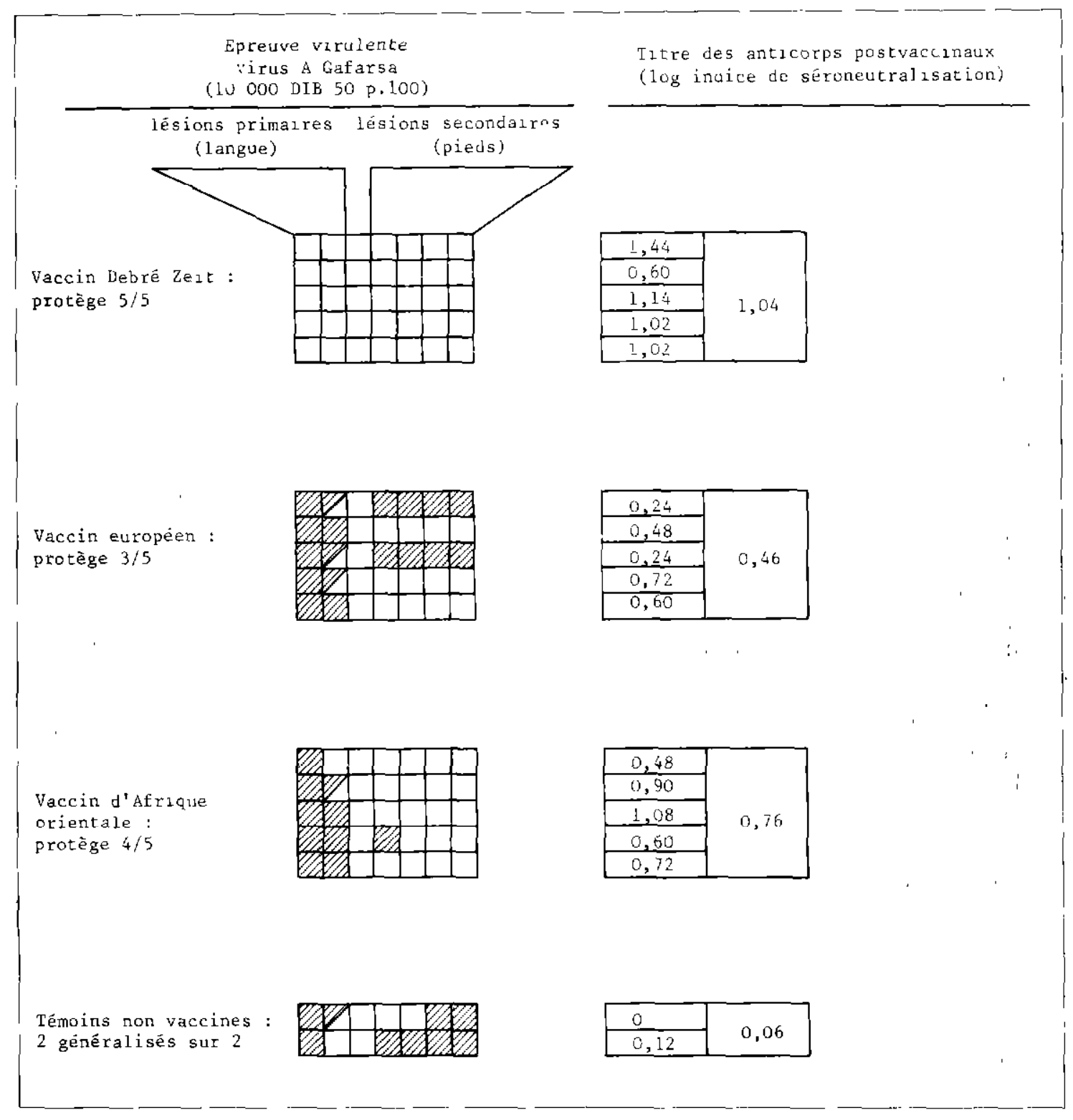

\section{B. Etude immunologique}

Le tableau $n^{\circ}$ II présente les résultats de la lecture de l'épreuve virulente des bovins avec 10000 doses infectieuses bovines 50 p. 100 de virus A Gafarsa et, pour chaque lot de bovins, la moyenne arithmétique des logarithmes des indices de séroneutralisation.

\section{DISCUSSION}

\section{A. Etude sérologique}

Comme toutes les autres souches éthiopiennes de type A (5) la souche A Gafarsa est sérologi* quement très différente de la souche européenne A5.

La souche A Gafarsa est probablement assez différente des autres souches A éthiopiennes à l'exception de la souche A Koka. Il faut préciser ici que la souche A Koka a été isolée en février 1974 après une absence totale pendant 4 années de foyers de ce type en Ethiopie. Au cours de l'année 1974, en revanche, l'épizootie de fièvre aphteuse de type A a sévi dans le centre de l'Ethiopie et il semble bien que la souche A Gafarsa, isolée en novembre 1974, corresponde bien à la même vague épizootique que la souche A Koka. 


\section{B Etude immunologique}

\section{1) Epreuve virulente des bovins}

En raison d'impératifs économiques, nous n'avons pas pu mettre en cuvre une méthode directe quantitative. La méthode utilisée est qualitative pour deux raisons :

- chaque bovin a été immunisé avec une dose fixe de vaccin et éprouvé avec une dose fixe de virus. Chaque animal ne donne qu'une seule réponse : protection ou absence de protection ;

- le nombre de bovins, limité à 5 dans chaque lot, ne permet pas de déduire un pourcentage de protection avec suffisamment de précision. En effet, pour 5 animaux protégés sur 5 éprouvés, comme dans le cas du vaccin expérimental de Debré Zeit, l'interprétation statistique (3), dans l'intervalle de confiance à 95 p. 100 généralement admis en biologie, indique que le pourcentage probable de protection de la population bovine varie entre 48 p. 100 et 100 p. 100.

De plus, cette épreuve est unilatérale car nous n'avons éprouvé les bovins que vis-à-vis de la souche A Gafarsa.

Malgré tout, on peut mettre en évidence l'insuffisance de couverture immunologique des vaccins commerciaux vis-à-vis de la souche A Gafarsa.

\section{2) Titrage des anticorps post-vaccinaux}

Le virus utilisé pour la séroneutralisation étant le virus A Gafarsa, le titre des anticorps post-vaccinaux des bovins vaccinés avec le vaccin expérimental de Debré Zeit est un titre homologue.
En revanche, les titres sériques des bovins des lots $\mathrm{n}^{\circ} 2$ et 3 sont hétérologues. On met en évidence aussi par cette méthode une nette différence entre les souches vaccinales exotiques et la souche locale A Gafarsa.

\section{CONCLUSION}

La souche A Gafarsa isolée en Ethiopie lors de l'épizootie de fièvre aphteuse de 1974 semble sérologiquement distincte des souches A éthiopiennes isolées dans ce pays avant 1971. De plus, elle est très différente sérologiquement de la souche européenne A5.

La couverture immunologique procurée par deux vaccins commerciaux importés d'Europe et d'Afrique orientale s'avère insuffisante pour protéger des bovins contre l'épreuve virulente avec la souche A Gafarsa. Nous admettons a priori la bonne qualité intrinsèque des vaccins importés, ce qui nous conduit à penser que ces échecs sont dus à une grande différence immunologique entre la souche éthiopienne A Gafarsa et les souches vaccinales exotiques. C'est ce que semble confirmer la relative faiblesse des taux d'anticorps post-vaccinaux hétérologues.

\section{REMERCIEMENTS}

Nous tenons à remercier: le Laboratoire Roger BELLON, B. P. de Villaines-les-Rochers, par Azay-le-Rideau, France, pour sa coopération et le matériel biologique qu'il nous a procuré.

Nous remercions également le Docteur J. FIKRE et le Docteur M. VIGIER, co-directeurs du Laboratoire de Debré ZEIT qui nous ont donné les moyens de réaliser cette expérimentation.

\section{SUMMARY}

Foot and mouth disease in Ethiopia : Serological and immunological study of FMDV type A

We have studied the strain $\mathbf{A}$ isolated in Gafarsa during the epizooty of 1974 in the center of Ethiopia.

The serology shows this strain is different of the others type A strains isolated in Ethiopia before 1971.

The immunology proves the imported vaccines from Europe and from East Africa don't give enough protection against our local strain A Gafarsa, probably because the strain Gafarsa is immunologically too different from the two exotic vaccinal strains. 


\section{RESUMEN}

La fiebre aftosa en Etiopia : estudio serologico

e inmunologico de una cepa de tipo $A$

Se presenta el estudio serologico e inmunologico de la cepa A Gafarsa, aislada en Etiopia durante la epizootia de 1974.

El estudio serologico da la prueba que esta cepa es diferente de las cepas aisladas durante las precedentes epizootias.

La proteccion inmunologica producida por dos vacunas exoticas es insuficiente para proteger los bovinos contra la cepa A Gafarsa.

\section{BIBLIOGRAPHIE}

1. BERSON (J. P.), COLSON (X.), FIKRE (J.), VIGIER (M.), ASSEFA (W. G.), GUERCHE (J.), BLANC (R.) et PRUNET (P.). Etude épizootologique de la fièvre aphteuse en Ethiopie (1969 à 1971). Bull. Off. int. Epiz., 1972, 77 (3-4) : 595-620.

2. FEDIDA (M.). Etude quantitative de l'état post vaccinal dans une virose animale, la fièvre aphteuse. Thèse Doct. Sci. Nat. Lyon, 1971.

3. GAYOT (G.), LUCAS (A.), DHENNIN (Mme L.) et DHENNIN (L.). Interprétation statistique de la méthode dite qualitative d'appréciation des vaccins antiaphteux. Bull. Acad, vét., 1965, 38 : 127-134.

4. MARTEL (J. L.). La fièvre aphteuse en Ethiopie.Distribution des sérotypes de virus aphteux. Rev. Elev. Méd. vét. Pays trop., 1974, 27 (2) : 169-175.

5. MARTEL (J. L.). Etude sérologique comparative des principales souches de virus aphteux isolées en Ethiopie de 1969 à 1974. Rev. Elev. Méd. vét. Pays trop., 1975, 28 (3) : 287-295.

6. Office International des Epizooties: Recommandations pour le contrôle des vaccins antiaphteux. Bull. Off. int. Epiz., 1972, 77 (9-10) : 1374. 